\title{
One-sample tests for a generalized Fréchet variance of a fuzzy random variable
}

\author{
Ana Belén Ramos-Guajardo • Ana Colubi • \\ Gil González-Rodríguez • María Ángeles Gil
}

Received: date / Accepted: date

\begin{abstract}
A procedure to test hypotheses about the population variance of a fuzzy random variable is analyzed. The procedure is based on the theory of UH-statistics. The variance is defined in terms of a general metric to quantify the variability of the fuzzy values about its (fuzzy) mean. An asymptotic one-sample test in a wide setting is developed and a bootstrap test, which is more suitable for small and moderate sample sizes, is also studied. Moreover, the power function of the asymptotic procedure through local alternatives is analyzed. Some simulations showing the empirical behavior and consistency of both tests are carried out. Finally, some illustrative examples of the practical application of the proposed tests are presented.
\end{abstract}

Keywords fuzzy random variable - support function · hypothesis testing · Fréchet variance $\cdot$ bootstrap techniques $\cdot \mathrm{UH}$-statistics

\section{Introduction}

Fuzzy random variables (FRVs for short) were introduced to model random mechanisms whose outcomes are associated with fuzzy sets (see [21]). As in the case of classical random variables, it is interesting to describe the distribution of an FRV by means of certain measures which summarize some of its characteristics, as the central tendency, the dispersion, etc.

A.B. Ramos-Guajardo · G. González-Rodríguez

European Centre for Soft Computing

C/ Gonzalo Gutiérrez Quirós s/n, 33600, Mieres-Asturias (Spain)

Tel.: +34-985-456545

Fax: +34-985-456699

E-mail: anabelen.ramos@softcomputing.es, gil.gonzalez@softcomputing.es

A. Colubi - M.A. Gil

Dpto. de Estadística, I.O y D.M.

C/ Calvo Sotelo s/n, 33007, Oviedo (Spain)

Tel.: +34-985-103356

Fax: +34-985-103356

E-mail: colubi@uniovi.es, magil@uniovi.es 
In this way, the fuzzy mean of an FRV has been introduced by Puri and Ralescu in [21] as a fuzzy-valued measure summarizing the distribtuion of the variable by means of its 'central' position. Regarding the one-sample test for the fuzzy mean, Körner [14] and Montenegro et al. [17] have developed the first asymptotic procedures.

Another important summary characteristic in Statistics is the 'variability' (variation or dispersion). The concept of variance considered in this paper is a Fréchet's one, that is, it is defined in terms of a squared-distance between variable values and the expected value (see, for instance, $[16,18,19]$ ). In other words, the variance corresponds to a 'squared error' associated with the summarization of the distribution through the fuzzy mean.

In some situations one can be interested in testing whether or not the variance of an FRV is equal to, greater than or lower than a given value. Lubiano et al. [16] have analyzed the problem of testing hypothesis for the variance of simple FRVs (i.e., those taking on a finite number of different values) in a particular class. The techniques used in [16] are based on large samples theory and an operational metric on the space of fuzzy numbers with compact support introduced by Bertoluzza et al. [2]. In this paper, the studies developed in [16] are extended to a wider class of non-necessarily simple FRVs and by using a generalized metric.

The convergence of the statistics are analyzed by employing some techniques based on the theory of UH-statistics [13]. Furthermore, taking inspiration on the bootstrap tests for the fuzzy mean of an FRV [10,12] some bootstrap techniques useful for small and moderated samples are studied.

The theoretical results developed in this context were mainly focussed on the significance level or type I error. The importance of the power function to establish the capability of a test is well-known, although often difficult to establish. In this respect, a way to analyze the power function by means of sequences of local alternatives converging to the null hypothesis as the sample size increases is presented here.

The rest of the paper is organized as follows. In Section 2 some preliminary concepts are introduced. The asymptotic and bootstrap tests are developed in Sections 3 and 4 respectively. The behavior under local alternatives of the asymptotic case is analyzed in Section 5. In addition, some simulations and some real examples are presented in Sections 6 and 7 in order to illustrate the empirical behavior of the tests. Finally, some concluding remarks and open problems are gathered in Section 8.

\section{Preliminary concepts}

Consider the $p$-dimensional Euclidean space $\mathbb{R}^{p}$ with the usual norm $\|\cdot\|_{p}$. Denote by $\mathcal{K}_{c}\left(\mathbb{R}^{p}\right)$ the class of nonempty compact convex subsets of $\mathbb{R}^{p}$ and by $\mathcal{F}_{c}\left(\mathbb{R}^{p}\right)$ the following class of upper semicontinuous functions of $\mathbb{R}^{p}$ (often referred to as the class of the compact convex fuzzy sets of $\mathbb{R}^{p}$ )

$$
\mathcal{F}_{c}\left(\mathbb{R}^{p}\right)=\left\{U: \mathbb{R}^{p} \rightarrow[0,1] \mid U_{\alpha} \in \mathcal{K}_{c}\left(\mathbb{R}^{p}\right) \text { for all } \alpha \in[0,1]\right\}
$$

where $U_{\alpha}$ denotes the $\alpha$-level of the fuzzy set $U$ (i.e. $U_{\alpha}=\left\{x \in \mathbb{R}^{p} \mid U(x) \geq \alpha\right\}$ ) for all $\alpha \in(0,1]$, and $U_{0}$ is the closure of the support of $U$. 
The space $\mathcal{F}_{c}\left(\mathbb{R}^{p}\right)$ can be naturally endowed with an inner composition law and a external one extending levelwise the Minkowski addition and the product by a scalar, that is,

$$
\begin{gathered}
(U+V)_{\alpha}=U_{\alpha}+V_{\alpha}=\left\{u+v: u \in U_{\alpha}, v \in V_{\alpha}\right\}, \\
(\lambda U)_{\alpha}=\lambda U_{\alpha}=\left\{\lambda u: u \in U_{\alpha}\right\}
\end{gathered}
$$

for all $U, V \in \mathcal{F}_{c}\left(\mathbb{R}^{p}\right), \lambda \in \mathbb{R}$ and $\alpha \in[0,1]$. These arithmetics agree with Zadeh's extension principle [22].

On the other hand, the lack of opposite element w.r.t. the Minkowski addition makes it sometimes interesting to consider the Hukuhara difference $U-{ }_{H} V$ of two fuzzy sets $U, V \in \mathcal{F}_{c}\left(\mathbb{R}^{p}\right)$, which is defined (if it exists) as the element $W \in \mathcal{F}_{c}\left(\mathbb{R}^{p}\right)$ such that $U=V+W$.

In order to take advantage of the properties of the classical statistical analysis in Hilbert spaces, the concept of the support function of a fuzzy set becomes a very useful tool. Given a fuzzy set $U \in \mathcal{F}_{c}\left(\mathbb{R}^{p}\right)[20]$, the support function of $U$ is defined as the function $s_{U}: \mathbb{S}^{p-1} \times[0,1] \rightarrow \mathbb{R}$ such that

$$
s_{U}(u, \alpha)=\sup _{w \in U_{\alpha}}\langle u, w\rangle
$$

for all $u \in \mathbb{S}^{p-1}$ and $\alpha \in[0,1]$, where $\mathbb{S}^{p-1}$ is the unit sphere in $\mathbb{R}^{p}$ (i.e. $\mathbb{S}^{p-1}=\{u \in$ $\left.\left.\mathbb{R}^{p} \mid\|u\|_{p}=1\right\}\right)$ and $\langle\cdot, \cdot\rangle$ denotes the inner product in $\mathbb{R}^{p}$.

A relevant feature of the support function is that it allows us to embed the space of fuzzy sets onto a cone of the Hilbert space of the square integrable functions $\mathcal{L}\left(\mathbb{S}^{p-1} \times\right.$ $[0,1])$ by means of the mapping $s: \mathcal{F}_{c}\left(\mathbb{R}^{p}\right) \longrightarrow \mathcal{L}\left(\mathbb{S}^{p-1} \times[0,1]\right)$ where $s(U)=s_{U}$. Furthermore, the support function preserves the semi-linear structure of $\mathcal{F}_{c}\left(\mathbb{R}^{p}\right)$, that is, if $U, V \in \mathcal{F}_{c}\left(\mathbb{R}^{p}\right), \lambda>0, s_{U+V}=s_{U}+s_{V}, s_{\lambda U}=\lambda s_{U}$, and if the Hukuhara difference $U-{ }_{H} V$ exists, it can be shown that $s_{U-H} V=s_{U}-s_{V}$.

By using this last concept and being inspired by [18] we can consider a generalized metric $D$ in $\mathcal{F}_{c}\left(\mathbb{R}^{p}\right)$ defined so that

$$
D(U, V)=\left\langle s_{U}-s_{V}, s_{U}-s_{V}\right\rangle=\left\|s_{U}-s_{V}\right\|^{2}
$$

for all $U, V \in \mathcal{F}_{c}\left(\mathbb{R}^{p}\right)$, where $\langle\cdot, \cdot\rangle$ represent a generic separate inner product on the Hilbert space $\mathcal{L}\left(\mathbb{S}^{p-1} \times[0,1]\right)$ and $\|\cdot\|$ is the corresponding norm. Thus each $D$ induces an isometry between $\mathcal{F}_{c}\left(\mathbb{R}^{p}\right)$ and the convex cone $s\left(\mathcal{F}_{c}\left(\mathbb{R}^{p}\right)\right) \subset \mathcal{L}\left(\mathbb{S}^{p-1} \times[0,1]\right)$. Special families of metrics of this type can be found, for instance, in $[2,8,18]$.

Given a probability space $(\Omega, \mathcal{A}, P)$, a fuzzy random variable (FRV for short) in Puri \& Ralescu's sense [21] is a mapping $\mathcal{X}: \Omega \rightarrow \mathcal{F}_{c}\left(\mathbb{R}^{p}\right)$ fulfilling that the $\alpha$-level mappings $\mathcal{X}_{\alpha}: \Omega \rightarrow \mathcal{K}_{c}\left(\mathbb{R}^{p}\right)$ (defined so that $\mathcal{X}_{\alpha}(w)=(\mathcal{X}(w))_{\alpha}$ for all $w \in \Omega$ ) are random sets (i.e., Borel-measurable mappings with the Borel $\sigma$-field generated by the topology associated with the well-known Hausdorff metric $d_{H}$ on $\mathcal{K}_{c}\left(\mathbb{R}^{p}\right)$ ). This definition is equivalent to say that an FRV is a Borel measurable mapping w.r.t. the most used $D$-type metrics (see [6] and [15]). The Borel measurability allows us to properly refer to the 'induced' distribution of an FRV, the independence of several FRVs, and so on.

In case that the FRV $\mathcal{X}$ satisfies that $\sup _{x \in \mathcal{X}_{0}}\|x\|_{p} \in L^{1}(\Omega, \mathcal{A}, P)$, the fuzzy expected value (or fuzzy mean) of $\mathcal{X}, E(\mathcal{X})$, is defined as the unique fuzzy set such that, for all $\alpha \in[0,1],(E(\mathcal{X}))_{\alpha}=$ Aumman's integral of the random set $\mathcal{X}_{\alpha}$ (see $\left.[1,21]\right)$. 
Moreover, if $E\left(\left(\sup _{x \in \mathcal{X}_{0}}\|x\|_{p}\right)^{2}\right)<\infty$, the dispersion of $\mathcal{X}$ can be quantified by means of the $D$-variance (or simply variance, inspired on [18]), which is defined in the Fréchet's sense (see [9]) as the real value $\sigma_{\mathcal{X}}^{2}=E(D(\mathcal{X}, E(\mathcal{X})))$, or, equivalently, in terms of the support function, $\sigma_{\mathcal{X}}^{2}=E\left(\left\langle s_{\mathcal{X}}-s_{E(\mathcal{X})}, s_{\mathcal{X}}-s_{E(\mathcal{X})}\right\rangle\right)$. It should be pointed out that the expected value in the Aumman's sense is a Frèchet expectation w.r.t. $D$, since

$$
E(D(\mathcal{X}, E(\mathcal{X})))=\inf _{U \in \mathcal{F}_{c}\left(\mathbb{R}^{p}\right)} E(D(\mathcal{X}, U)) .
$$

For the hypothesis testing problems, it is useful to define some sample moments of the fuzzy sets. Consider a simple random sample of $n$ independent observations, $\mathcal{X}_{1}, \ldots, \mathcal{X}_{n}$, from an FRV $\mathcal{X}$. The fuzzy sample mean of $\left\{\mathcal{X}_{i}\right\}_{i=1}^{n}$ is given by $\overline{\mathcal{X}}_{n}=$ $(1 / n) \sum_{i=1}^{n} \mathcal{X}_{i}$. The semilinearity properties of the support function imply that $s \overline{\mathcal{X}}_{n}=$ $\overline{s \mathcal{X}}$.

In the same way, it is possible to define the sample variance of $\left\{\mathcal{X}_{i}\right\}_{i=1}^{n}$ as $\widehat{\sigma}_{\mathcal{X}}^{2}=$ $(1 / n) \sum_{i=1}^{n} D\left(\mathcal{X}_{i}, \overline{\mathcal{X}}_{n}\right)$ although considering $\widehat{S}_{\mathcal{X}}^{2}=(1 /(n-1)) \sum_{i=1}^{n} D\left(\mathcal{X}_{i}, \overline{\mathcal{X}}_{n}\right)$ would be even more usual because it is an unbiased and consistent estimator of the population variance (see $[18]$ ).

\section{Asymptotic tests}

The theory of UH-statistics (or statistics in a Hilbert space) was developed in [13]. Given $X_{1}, \ldots, X_{n}$ independent and identically distributed RVs taking on values in a measurable space, and an arbitrary separable real Hilbert space $H$ with the inner product $\langle\cdot, \cdot\rangle_{H}$ and associated norm $\|\cdot\|_{H}$, a $U H-$ statistic $U_{n}$ is defined by

$$
U_{n}=\left(\begin{array}{c}
n \\
m
\end{array}\right)^{-1} \sum_{1 \leq i_{1}<\ldots<i_{m} \leq n} \Phi\left(X_{i_{1}}, \ldots, X_{i_{m}}\right)
$$

where $\Phi: X^{m} \longrightarrow H$ is a symmetric kernel.

Consider now an FRV $\mathcal{X}$ so that $E\left(\left(\sup _{x \in \mathcal{X}_{0}}\|x\|_{p}\right)^{2}\right)<\infty$ and a simple random sample $\mathcal{X}_{1}, \ldots, \mathcal{X}_{n}$ from $\mathcal{X}$. Then, $s_{\mathcal{X}_{1}}, \ldots, s \mathcal{X}_{n}$ are independent and identically distributed RVs taking on values in the measurable space $\left(\mathcal{L}\left(\mathbb{S}^{p-1} \times[0,1]\right), \beta\right)$ (where $\beta$ is the Borel $\sigma$-field associated with the metric $D)$. On the other hand, consider as $H$ the Hilbert space $\mathbb{R}$ with the associated norm $\|\cdot\|_{\mathbb{R}}$ and define the UH-statistic $U_{n}=\left(\begin{array}{c}n \\ 2\end{array}\right)^{-1} \sum_{1 \leq i<j \leq n} \Phi\left(s_{\mathcal{X}_{i}}, s_{\mathcal{X}_{j}}\right)$ where the kernel $\Phi: \mathcal{L}\left(\mathbb{S}^{p-1} \times[0,1]\right) \times \mathcal{L}\left(\mathbb{S}^{p-1} \times\right.$ $[0,1]) \longrightarrow \mathbb{R}$ is such that $\Phi\left(s_{\mathcal{X}_{i}}, s_{\mathcal{X}_{j}}\right)=\frac{1}{2}\left\|s_{\mathcal{X}_{i}}-s_{\mathcal{X}_{j}}\right\|^{2}$.

Proposition 1 Under the previous conditions $U_{n}=\widehat{S}_{\mathcal{X}}^{2}$.

Proof. $U_{n}$ can be written as

$$
\begin{aligned}
U_{n}= & \left(\begin{array}{l}
n \\
2
\end{array}\right)^{-1} \sum_{1 \leq i<j \leq n} \frac{1}{2}\left\|s \mathcal{X}_{i}-s \mathcal{X}_{j}\right\|^{2}=\frac{1}{n(n-1)} \sum_{1 \leq i<j \leq n}\left\|s \mathcal{X}_{i}-s \mathcal{X}_{j}\right\|^{2} \\
= & \frac{1}{2(n-1)} \sum_{i}\left\|s \mathcal{X}_{i}-s \overline{\mathcal{X}}_{n}\right\|^{2}+\frac{1}{2(n-1)} \sum_{j}\left\|s \mathcal{X}_{j}-s \overline{\mathcal{X}}_{n}\right\|^{2} \\
& -\frac{2}{2 n(n-1)} \sum_{i} \sum_{j}\left\langle s \mathcal{X}_{i}-s \overline{\mathcal{X}}_{n}, s \overline{\mathcal{X}}_{n}-s \mathcal{X}_{j}\right\rangle
\end{aligned}
$$


Since the last term vanishes, we have that $U_{n}=\widehat{S}_{\mathcal{X}}^{2}$.

Furthermore, one can compute the variance of $\widehat{S}_{\mathcal{X}}^{2}$ by using the following result:

Lemma 1 [13] If $U_{n}=\left(\begin{array}{c}n \\ m\end{array}\right)^{-1} \sum_{1 \leq i_{1}<\ldots<i_{m} \leq n} \Phi\left(X_{i_{1}}, \ldots, X_{i_{m}}\right)$ is an UH-statistic verifying the condition $E\left(\Phi^{2}\left(X_{i_{1}}, \ldots, X_{i_{m}}\right)\right)<\infty$, then

$$
\sigma_{U_{n}}^{2}=\left(\begin{array}{c}
n \\
m
\end{array}\right)^{-1} \sum_{k=1}^{m}\left(\begin{array}{c}
m \\
k
\end{array}\right)\left(\begin{array}{c}
n-m \\
m-k
\end{array}\right) \zeta_{k}
$$

where $\zeta_{k}=\sigma_{\Phi_{k}\left(X_{1}, \ldots, X_{k}\right)}^{2}$ and $\Phi_{k}$ is such that

$$
\Phi_{k}\left(x_{1}, \ldots, x_{k}\right)=E\left(\Phi\left(X_{1}, \ldots X_{n}\right) \mid X_{1}=x_{1}, \ldots, X_{k}=x_{k}\right) .
$$

Proposition 2 Under the previous conditions

$$
\sigma_{\widehat{S}_{\mathcal{X}}^{2}}^{2}=\frac{\sigma_{D(\mathcal{X}, E(\mathcal{X}))}^{2}}{n}+\frac{2 \sigma_{\mathcal{X}}^{4}}{n(n-1)} .
$$

Proof. Using the Lemma 1 with $\Phi\left(s_{\mathcal{X}_{i}}, s_{\mathcal{X}_{j}}\right)=\frac{1}{2}\left\|s_{\mathcal{X}_{i}}-s_{\mathcal{X}_{j}}\right\|^{2}$ we have that,

$$
\sigma_{\widehat{S}_{\mathcal{X}}^{2}}^{2}=\left(\begin{array}{l}
n \\
2
\end{array}\right)^{-1}\left[\left(\begin{array}{l}
2 \\
1
\end{array}\right)\left(\begin{array}{l}
n-2 \\
2-1
\end{array}\right) \zeta_{1}+\left(\begin{array}{l}
2 \\
2
\end{array}\right)\left(\begin{array}{l}
n-2 \\
2-2
\end{array}\right) \zeta_{2}\right]
$$

Subtracting and adding $s_{E(\mathcal{X})}$ results that

$$
\begin{gathered}
\Phi_{i}\left(s \mathcal{X}_{i}\right)=\frac{1}{2}\left\|s \mathcal{X}_{i}-s_{E(\mathcal{X})}\right\|^{2}+\frac{1}{2} \sigma_{\mathcal{X}}^{2} \\
\Phi_{(i, j)}\left(s_{\mathcal{X}_{i}}, s_{\mathcal{X}_{j}}\right)=\frac{1}{2}\left\|s_{\mathcal{X}_{i}}-s_{E(\mathcal{X})}\right\|^{2}+\frac{1}{2}\left\|s_{E(\mathcal{X})}-s \mathcal{X}_{j}\right\|^{2}+\left\langle s_{\mathcal{X}_{i}}-s_{E(\mathcal{X})}, s_{E(\mathcal{X})}-s_{\mathcal{X}_{j}}\right\rangle
\end{gathered}
$$

Simplifying terms by using properties of the inner product and the support function it is verified that $\zeta_{1}=\sigma_{\Phi_{i}\left(s_{\mathcal{X}}\right)}^{2}=\frac{\sigma_{D(\mathcal{X}, E(\mathcal{X}))}^{2}}{4}$ and $\zeta_{2}=\frac{1}{4}\left(2 E\left(D^{2}(\mathcal{X}, E(\mathcal{X}))\right)+6 \sigma_{\mathcal{X}}^{4}\right.$. Therefore $\sigma_{\widehat{S}_{\mathcal{X}}^{2}}^{2}=\left(\begin{array}{l}n \\ 2\end{array}\right)^{-1}\left(2(n-2) \zeta_{1}+\zeta_{2}\right)=\frac{\sigma_{D(\mathcal{X}, E(\mathcal{X}))}^{2}}{n}+\frac{\sigma_{\mathcal{X}}^{2}}{n(n-1)}$.

On the other hand, the studies in [5] and [13] show that the UH-statistics satisfy the CLT. Suppose that $X_{1}, \ldots, X_{n}$ are independent and identically distributed RVs taking on values in a measurable space, and that $U_{n}$ is defined as in (1). If $E\left(\Phi^{2}\left(X_{i_{1}}, \ldots, X_{i_{m}}\right)\right)<\infty$, then $U_{n}$ converges in law to a normal distribution with the same mean and variance than $U_{n}$ as $n$ tends to $\infty$.

The condition $E\left(\frac{1}{4}\left\|s \mathcal{X}_{i}-s \mathcal{X}_{j}\right\|^{4}\right)<\infty$ is equivalent to $E\left(\|s \mathcal{X}\|^{4}\right)<\infty$. Then, consider the statistic

$$
\widetilde{Z}_{n}=\frac{\widehat{S}_{\mathcal{X}}^{2}-\sigma_{\mathcal{X}}^{2}}{\sqrt{\frac{\sigma_{D(\mathcal{X}, E(\mathcal{X}))}^{2}}{n}+\frac{2 \sigma_{\mathcal{X}}^{4}}{n(n-1)}}} .
$$

Thus, by applying the CLT for UH-statistics, the next theorem is easy to be derived: 
Theorem 1 Let $\mathcal{X}$ be an $F R V$ and $\mathcal{X}_{1}, \ldots, \mathcal{X}_{n}$ be a simple random sample from $\mathcal{X}$. If $E\left(\|s \mathcal{X}\|^{4}\right)<\infty$, then $\widetilde{Z}_{n}$ converges in law to a standard normal distribution.

The aim of this work is to test the null hypothesis $H_{0}: \sigma_{\mathcal{X}}^{2}=\sigma_{0}^{2}$ versus $H_{1}: \sigma_{\mathcal{X}}^{2} \neq$ $\sigma_{0}^{2}$ or, equivalently, testing $H_{0}: E(D(\mathcal{X}, E(\mathcal{X})))=\sigma_{0}^{2}$ versus $H_{1}: E(D(\mathcal{X}, E(\mathcal{X}))) \neq$ $\sigma_{0}^{2}$ for a given $\sigma_{0} \in \mathbb{R}^{+}$. In the same way, the one-sided tests for the variance of an FRV are considered, that is, the null hypotheses $H_{0}: \sigma_{\mathcal{X}}^{2} \leq \sigma_{0}^{2}$ and $H_{0}: \sigma_{\mathcal{X}}^{2} \geq \sigma_{0}^{2}$ are also to be tested.

In order to face this problem we are now going to analyze the asymptotic behavior of the following basic statistic:

$$
Z_{n}=\frac{\sqrt{n}\left(\widehat{S}_{\mathcal{X}}^{2}-\sigma_{\mathcal{X}}^{2}\right)}{\sqrt{\sigma_{D(\mathcal{X}, E(\mathcal{X}))}^{2}}}
$$

Theorem 2 If $E\left(\|s \mathcal{X}\|^{4}\right)<\infty, Z_{n}$ converges in law to a distribution $\mathcal{N}(0,1)$.

Proof. From Theorem 1 we have that $\widetilde{Z}_{n}$ converges in law to a variable $\mathcal{N}(0,1)$. Since $Z_{n}$ can be written as $Z_{n}=\widetilde{Z}_{n} \cdot A_{n}$, and

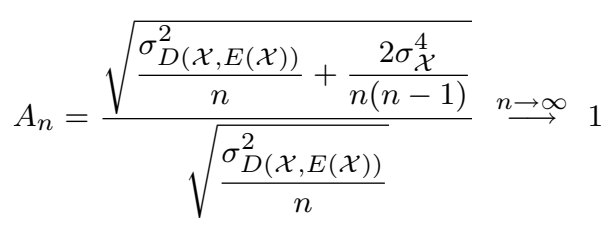

the result is obtained.

The denominator of $Z_{n}$ is a population value, so it is convenient to estimate it in order to define later the test statistic. The proposed estimator is

$$
\widehat{\sigma}_{D\left(\mathcal{X}, \overline{\mathcal{X}}_{n}\right)}^{2}=\frac{1}{n} \sum_{i=1}^{n}\left(D\left(\mathcal{X}_{i}, \overline{\mathcal{X}}_{n}\right)-\frac{1}{n} \sum_{i=1}^{n} D\left(\mathcal{X}_{i}, \overline{\mathcal{X}}_{n}\right)\right)^{2} .
$$

This estimator is examined in the following proposition:

Proposition 3 Let $\mathcal{X}$ be an $F R V$ and $\mathcal{X}_{1}, \ldots, \mathcal{X}_{n}$ a simple random sample obtained from $\mathcal{X}$. If $E\left(\|s \mathcal{X}\|^{4}\right)<\infty$, then $\left\{\widehat{\sigma}_{D\left(\mathcal{X}, \overline{\mathcal{X}}_{n}\right)}^{2}\right\}_{n}$ is a strongly consistent sequence of estimators of $\sigma_{D(\mathcal{X}, E(\mathcal{X}))}^{2}$.

Proof. In a first step, it will be proved that the sequence of estimators defined for all $n \in \mathbb{N}$ as

$$
A_{n}=\frac{1}{n} \sum_{i=1}^{n}\left(D\left(\mathcal{X}_{i}, E(\mathcal{X})\right)-\frac{1}{n} \sum_{i=1}^{n} D\left(\mathcal{X}_{i}, E(\mathcal{X})\right)\right)^{2}
$$

is strongly consistent with $\sigma_{D(\mathcal{X}, E(\mathcal{X}))}^{2}$. Indeed, $A_{n}$ can be expressed as

$$
\begin{gathered}
A_{n}=\frac{1}{n} \sum_{i=1}^{n}\left(D\left(\mathcal{X}_{i}, E(\mathcal{X})\right)-E(D(\mathcal{X}, E(\mathcal{X})))\right)^{2} \\
-\left(E(D(\mathcal{X}, E(\mathcal{X})))-\frac{1}{n} \sum_{i=1}^{n} D\left(\mathcal{X}_{i}, E(\mathcal{X})\right)\right)^{2} .
\end{gathered}
$$

Note that the first term of that expansion is the sample variance of the real-valued RVs $\left\{D\left(\mathcal{X}_{i}, E(\mathcal{X})\right)\right\}_{i=1}^{n}$ which converges almost surely to $\sigma_{D(\mathcal{X}, E(\mathcal{X}))}^{2}$. The strong law of 
large numbers guarantees that the second term converges almost surely to 0 . Therefore $\left\{A_{n}\right\}_{n} \stackrel{a . s}{\longrightarrow} \sigma_{D(\mathcal{X}, E(\mathcal{X}))}^{2}$. In a second step, it will be proved that $\left(\widehat{\sigma}_{D\left(\mathcal{X}, \overline{\mathcal{X}}_{n}\right)}^{2}-A_{n}\right) \stackrel{\text { a.s. }}{\longrightarrow}$

0 . Note first that by operating in the expression of $\widehat{\sigma}_{\mathcal{X}}^{2}$, it results that

$$
\widehat{\sigma}_{D\left(\mathcal{X}, \overline{\mathcal{X}}_{n}\right)}^{2}=A_{n}-\frac{4}{n} \sum_{i=1}^{n}\left(\left\langle s \mathcal{X}_{i}-s_{\overline{\mathcal{X}}_{n}}, s_{\mathcal{X}_{i}}-s_{E(\mathcal{X})}\right\rangle\left\langle s_{\mathcal{X}_{i}}-s_{\overline{\mathcal{X}}_{n}}, s_{\overline{\mathcal{X}}_{n}}-s_{E(\mathcal{X})}\right\rangle\right) .
$$

Thus, it is enough to prove that the second term of the last expression (denoted by $B_{n}$ ) converges to 0 as $n \rightarrow \infty$. $B_{n}$ can be decomposed as follows:

$$
\begin{aligned}
& B_{n}=-\frac{4}{n} \sum_{i=1}^{n}\left(\left\langle s_{\mathcal{X}_{i}}, s_{\mathcal{X}_{i}}\right\rangle\left\langle s_{\mathcal{X}_{i}}, s_{\overline{\mathcal{X}}_{n}}\right\rangle-\left\langle s_{\mathcal{X}_{i}}, s_{\mathcal{X}_{i}}\right\rangle\left\langle s_{\mathcal{X}_{i}}, s_{E(\mathcal{X})}\right\rangle\right) \\
& -\frac{4}{n} \sum_{i=1}^{n}\left(\left\langle s \mathcal{X}_{i}, s \mathcal{X}_{i}\right\rangle\left\langle s \overline{\mathcal{X}}_{n}, s_{E(\mathcal{X})}\right\rangle-\left\langle s \mathcal{X}_{i}, s \mathcal{X}_{i}\right\rangle\left\langle s \overline{\mathcal{X}}_{n}, s \overline{\mathcal{X}}_{n}\right\rangle\right) \\
& -\frac{4}{n} \sum_{i=1}^{n}\left(\left\langle s_{\mathcal{X}_{i}}, s_{E(\mathcal{X})}\right\rangle\left\langle s_{\mathcal{X}_{i}}, s_{E(\mathcal{X})}\right\rangle-\left\langle s_{\mathcal{X}_{i}}, s_{E(\mathcal{X})}\right\rangle\left\langle s_{\mathcal{X}_{i}}, s_{\overline{\mathcal{X}}_{n}}\right\rangle\right) \\
& -\frac{4}{n} \sum_{i=1}^{n}\left(\left\langle s \overline{\mathcal{X}}_{n}, s \mathcal{X}_{i}\right\rangle\left\langle s \overline{\mathcal{X}}_{n}, s \overline{\mathcal{X}}_{n}\right\rangle-\left\langle s \overline{\mathcal{X}}_{n}, s \mathcal{X}_{i}\right\rangle\left\langle s \overline{\mathcal{X}}_{n}, s_{E(\mathcal{X})}\right\rangle\right) \\
& -\frac{4}{n} \sum_{i=1}^{n}\left(\left\langle s \overline{\mathcal{X}}_{n}, s \mathcal{X}_{i}\right\rangle\left\langle s \mathcal{X}_{i}, s_{E(\mathcal{X})}\right\rangle-\left\langle s \overline{\mathcal{X}}_{n}, s \mathcal{X}_{i}\right\rangle\left\langle s \mathcal{X}_{i}, s_{\overline{\mathcal{X}}_{n}}\right\rangle\right) \\
& -\frac{4}{n} \sum_{i=1}^{n}\left(\left\langle s_{\mathcal{X}_{i}}, s_{E(\mathcal{X})}\right\rangle\left\langle s_{\overline{\mathcal{X}}_{n}}, s_{\overline{\mathcal{X}}_{n}}\right\rangle-\left\langle s_{\mathcal{X}_{i}}, s_{E(\mathcal{X})}\right\rangle\left\langle s_{\overline{\mathcal{X}}_{n}}, s_{E(\mathcal{X})}\right\rangle\right) \\
& -\frac{4}{n} \sum_{i=1}^{n}\left(\left\langle s_{\overline{\mathcal{X}}_{n}}, s_{E(\mathcal{X})}\right\rangle\left\langle s_{\mathcal{X}_{i}}, s_{\overline{\mathcal{X}}_{n}}\right\rangle-\left\langle s_{\overline{\mathcal{X}}_{n}}, s_{E(\mathcal{X})}\right\rangle\left\langle s_{\mathcal{X}_{i}}, s_{E(\mathcal{X})}\right\rangle\right) \\
& -\frac{4}{n} \sum_{i=1}^{n}\left(\left\langle s \overline{\mathcal{X}}_{n}, s_{E(\mathcal{X})}\right\rangle_{K}\left\langle s \overline{\mathcal{X}}_{n}, s_{E(\mathcal{X})}\right\rangle-\left\langle s \overline{\mathcal{X}}_{n}, s_{E(\mathcal{X})}\right\rangle\left\langle s \overline{\mathcal{X}}_{n}, s \overline{\mathcal{X}}_{n}\right\rangle\right)
\end{aligned}
$$

From the triangle and Cauchy-Schwarz inequalities we have that

$$
\begin{gathered}
\left|-\frac{4}{n} \sum_{i=1}^{n}\left(\left\langle s \mathcal{X}_{i}, s \mathcal{X}_{i}\right\rangle\left\langle s \mathcal{X}_{i}, s \overline{\mathcal{X}}_{n}\right\rangle-\left\langle s \mathcal{X}_{i}, s \mathcal{X}_{i}\right\rangle\left\langle s \mathcal{X}_{i}, s_{E(\mathcal{X})}\right\rangle\right)\right| \\
\leq \frac{4}{n} \sum_{i=1}^{n}\left\|s_{\mathcal{X}_{i}}\right\|^{3}\left\|s \overline{\mathcal{X}}_{n}-s_{E(\mathcal{X})}\right\| .
\end{gathered}
$$

The second and the fifth terms can be also upper bounded in a similar way. The condition $E\left(\|s \mathcal{X}\|^{4}\right)<\infty$ implies that $\left\|s_{E(\mathcal{X})}\right\|^{4}<\infty$ and, therefore, the moments of lower order are also finite. In addition, $s_{\overline{\mathcal{X}}_{n}} \stackrel{a . s}{\longrightarrow} s_{E(\mathcal{X})}$, so all the terms converges to 0 as $n \rightarrow \infty$ and $\widehat{\sigma}_{D\left(\mathcal{X}, \overline{\mathcal{X}}_{n}\right)}^{2}-A_{n} \stackrel{a . s}{\longrightarrow} 0$.

Hereafter, in order to carry out the proposed tests the statistic $T_{n}=\frac{\sqrt{n}\left(\widehat{S}_{\mathcal{X}}^{2}-\sigma_{0}^{2}\right)}{\sqrt{\widehat{\sigma}_{D\left(\mathcal{X}, \overline{\mathcal{X}}_{n}\right)}^{2}}}$ is considered. On this basis, the following asymptotic procedure is presented: 
Theorem 3 Under the conditions in the previous theorems. Let $\alpha \in[0,1]$ :

a) Two-sided test. The significance level of the test that rejects $H_{0}: \sigma_{\mathcal{X}}^{2}=\sigma_{0}^{2}$ against $H_{1}: \sigma_{\mathcal{X}}^{2} \neq \sigma_{0}^{2}$ whenever $\left|T_{n}\right|>z_{1-\alpha / 2}$, where $z_{1-\alpha / 2}$ is the $[1-\alpha / 2]$-quantile of the distribution $N(0,1)$ converges to $\alpha$. The p-value of this test is approximately given by $p=2\left[1-\Phi\left(\left|T_{n}\right|\right)\right]$, where $\Phi$ is the cumulative distribution function of a $\mathcal{N}(0,1) R V$.

b) One-sided tests

(i) The significance level of the test that rejects $H_{0}: \sigma_{\mathcal{X}}^{2} \geq \sigma_{0}^{2}$ against $H_{1}: \sigma_{\mathcal{X}}^{2}<$ $\sigma_{0}^{2}$ whenever $T_{n}<z_{\alpha}$, where $z_{\alpha}$ is the $\alpha$-quantile of the distribution $N(0,1)$ converges to $\alpha$. The p-value of this test is approximately given by $p=\Phi\left(T_{n}\right)$.

(ii) The significance level of the test that rejects $H_{0}: \sigma_{\mathcal{X}}^{2} \leq \sigma_{0}^{2}$ against $H_{1}: \sigma_{\mathcal{X}}^{2}>\sigma_{0}^{2}$ whenever $T_{n}>z_{1-\alpha}$, where $z_{1-\alpha}$ is the $(1-\alpha)$-quantile of the distribution $N(0,1)$ converges to $\alpha$. The $p$-value of this test is approximately given by $p=$ $1-\Phi\left(T_{n}\right)$.

Remark 1 The classical statistic used to test the variance of a real-valued RV is based on the quotient $\widehat{S}_{\mathcal{X}}^{2} / \sigma_{\mathcal{X}}^{2}$. By the convergence of types (see [4]), there can be only one possible limit type and essentially one possible sequence of norming constants. Applying this result to the estimator $\widehat{S}_{\mathcal{X}}^{2}$, we have that the classical statistic (conveniently normalized) and $T_{n}$ are essentially the same.

Specifically, the classical statistic used to test the variance of a real normal variable has a $\chi_{n-1}^{2}$ distribution. It is known that $\widetilde{T}_{n}=\frac{\sqrt{n}\left(\widehat{S}_{X}^{2}-\sigma_{X}^{2}\right)}{\sigma_{X}^{2} \sqrt{2}}$, converges to a $\mathcal{N}(0,1)$. The denominator of the previous statistic is equal to the $T_{n}$ one when a real variable $\mathcal{N}(\mu, \sigma)$ is considered. Therefore $\widetilde{T}_{n}=T_{n}$ in the real-valued normal case.

\section{Bootstrap tests}

In Montenegro et al. [17], Gil et al. [10] and González-Rodríguez et al. [12], the application of bootstrap techniques to test the fuzzy mean of an FRV provides better results than the asymptotic ones. In this section analogous results about the test for the variance of an FRV are proved.

Suppose that $\mathcal{X}$ is an FRV defined on the probability space $(\Omega, \mathcal{A}, P)$, and that $n$ independent FRVs $\left\{\mathcal{X}_{1}, \ldots, \mathcal{X}_{n}\right\}$ distributed as $\mathcal{X}$ are considered. In addition, let $\left\{\mathcal{X}_{i}^{*}\right\}_{i=1}^{n}$ be a bootstrap sample from $\left\{\mathcal{X}_{i}\right\}_{i=1}^{n}$. Let

$$
T_{n}^{1^{*}}=\frac{\frac{\sigma_{\mathcal{X}}^{2}}{\widehat{S}_{\mathcal{X}}^{2}} \sqrt{n}\left(\widehat{S}_{\mathcal{X}^{*}}^{2}-\widehat{S}_{\mathcal{X}}^{2}\right)}{\sqrt{\widehat{\sigma}_{D\left(\mathcal{X}, \overline{\mathcal{X}}_{n}\right)}^{2}}} .
$$

where $\widehat{S}_{\mathcal{X}^{*}}^{2}=\frac{1}{n-1} \sum_{i=1}^{n} D\left(\mathcal{X}_{i}^{*}, \overline{\mathcal{X}}_{n}^{*}\right)$. Therefore, the following theorem can be stated:

Theorem 4 Under the previous conditions, if $E\left(\|s \mathcal{X}\|^{4}\right)<\infty$, then

$$
T_{n}^{1^{*}} \stackrel{\mathcal{L}}{\longrightarrow} \mathcal{N}(0,1)
$$


Proof. By applying the result in Bickel and Freedman [3] for U-statistics, it can be verified that $\sqrt{n}\left(\widehat{S}_{\mathcal{X}^{*}}^{2}-\widehat{S}_{\mathcal{X}}^{2}\right)$ converges to the same distribution than $\sqrt{n}\left(\widehat{S}_{\mathcal{X}}^{2}-\sigma_{\mathcal{X}}^{2}\right)$. From Theorem 2 we have that the last expression converges to a random variable $\mathcal{N}\left(0, \sigma_{D(\mathcal{X}, E(\mathcal{X}))}^{2}\right)$. Moreover, the convergence of the denominator of $T_{n}^{1^{*}}$ is given in the Proposition 3. Thus, taking into account that $\sigma_{\mathcal{X}}^{2} / \widehat{S}_{\mathcal{X}}^{2} \stackrel{a . s}{\longrightarrow} 1$, we conclude that

$$
T_{n}^{1^{*}} \stackrel{\mathcal{L}}{\longrightarrow} \mathcal{N}(0,1)
$$

Consider now the statistic $T_{n}^{1}=\sqrt{n}\left(S_{\mathcal{X}}^{2}-\sigma_{0}^{2}\right)$. As a consequence of the previous result, the following theorem is concluded:

Theorem 5 Under the conditions in Theorem 4. Let $\alpha \in[0,1]$ :

a) Two-sided test. The significance level of the test that rejects $H_{0}: \sigma_{\mathcal{X}}^{2}=\sigma_{0}^{2}$ against $H_{1}: \sigma_{\mathcal{X}}^{2} \neq \sigma_{0}^{2}$ whenever $\left|T_{n}^{1}\right|>z_{(1-\alpha / 2)}$, where $z_{(1-\alpha) / 2}$ is the $(1-\alpha / 2)$-quantile of the distribución of $T_{n}^{1^{*}}$, converges to $\alpha$.

b) One-sided tests

(i) The significance level of the test that rejects $H_{0}: \sigma_{\mathcal{X}}^{2} \geq \sigma_{0}^{2}$ against $H_{1}: \sigma_{\mathcal{X}}^{2}<\sigma_{0}^{2}$ whenever $T_{n}^{1}<z_{\alpha}$, where $z_{\alpha}$ is the $\alpha$-quantile of the distribution of $T_{n}^{1^{*}}$, converges to $\alpha$.

(ii) The significance level of the test that rejects $H_{0}: \sigma_{\mathcal{X}}^{2} \leq \sigma_{0}^{2}$ against $H_{1}: \sigma_{\mathcal{X}}^{2}>$ $\sigma_{0}^{2}$ whenever $T_{n}^{1}>z_{(1-\alpha)}$, where $z_{1-\alpha}$ is the $(1-\alpha)$-quantile of the distribution of $T_{n}^{1^{*}}$, converges to $\alpha$.

Remark 2 In practice the distribution of $T_{n}^{1^{*}}$ is unknown. In order to overcome this problem, the Montecarlo's approximation is employed as usual.

Moreover, it is possible to employ an alternative bootstrap procedure which consist on reestimate $\widehat{\sigma}_{D\left(\mathcal{X}, \overline{\mathcal{X}}_{n}\right)}^{2}$ by means of

$$
\frac{\sigma_{0}^{4}}{\widehat{S}_{\mathcal{X}}^{4}}\left(\frac{1}{n} \sum_{i=1}^{n}\left(D\left(\mathcal{X}_{i}^{*}, \overline{\mathcal{X}}_{n}^{*}\right)-\widehat{\sigma}_{\mathcal{X}^{*}}^{2}\right)^{2}\right) .
$$

Thus, consider the following bootstrap statistic

$$
T_{n}^{2^{*}}=\frac{\sqrt{n}\left(\widehat{S}_{\mathcal{X}^{*}}^{2}-\widehat{S}_{\mathcal{X}}^{2}\right)}{\sqrt{\frac{1}{n} \sum_{i=1}^{n}\left(D\left(\mathcal{X}_{i}^{*}, \overline{\mathcal{X}}_{n}^{*}\right)-\widehat{\sigma}_{\mathcal{X}^{*}}^{2}\right)^{2}}}
$$

as an approximation of the distribution of $T_{n}$ under the worse situation under $H_{0}$. Finally, use the Montecarlo method to approximate the unknown distribution of $T_{n}^{2^{*}}$.

The following bootstrap testing algorithm is then proposed:

Step 1. Choose a simple random sample $\left\{\mathcal{X}_{1}, \ldots \mathcal{X}_{n}\right\}$ of $n$ independent FRVs and identically distributed as the FRV $\mathcal{X}$.

Step 2. Compute for this sample the value of the statistic $T=T_{n}^{1}$.

Step 3. Obtain a sample $\left\{\mathcal{X}_{i}^{*}\right\}_{i=1}^{n}$ of $n$ independent and identically distributed FRVs from the bootstrap population $\left\{\mathcal{X}_{i}\right\}_{i=1}^{n}$ and compute the value of the bootstrap statistic $T_{n}^{1^{*}}$. 
Step 4. Repeat Step 3 a large number $B$ of times to get a set of $B$ values of the bootstrap estimator, denoted by $\left\{T_{1}^{*}, \ldots, T_{B}^{*}\right\}$.

Step 5. Compute the bootstrap $p$-value:

1. In the two-sided case, the approximate $p$-value is given by the proportion of values in $\left\{T_{1}^{*}, \ldots, T_{B}^{*}\right\}$ whose modulus is greater that the modulus of $\mathrm{T}$.

2. To test $H_{0}: \sigma_{\mathcal{X}}^{2} \geq \sigma_{0}^{2}$ against $H_{1}: \sigma_{\mathcal{X}}^{2}<\sigma_{0}^{2} \quad$ (or $H_{0}: \sigma_{\mathcal{X}}^{2} \leq \sigma_{0}^{2}$ against $H_{1}: \sigma_{\mathcal{X}}^{2}>\sigma_{0}^{2}$, respectively) the approximate $p$-value is given by the proportion of values in $\left\{T_{1}^{*}, \ldots, T_{B}^{*}\right\}$ whose value is smaller than (or greater than) $\mathrm{T}$.

In the second bootstrap approximation, Steps 2 and 3 are modified:

Step 2.* By using the initial sample, compute the value of the statistic $T=T_{n}$.

Step 3.* Obtain a sample of $n$ independent and identically distributed FRVs from the bootstrap population $\left\{\mathcal{X}_{i}\right\}_{i=1}^{n}$ and compute the value of the bootstrap statistic $T_{n}^{2^{*}}$.

\section{Power analysis (local alternatives)}

The analysis of the power function in order to establish the capability of a given test is a very difficult task in most of cases. A suitable way to carry out this analysis is through the study of the power function under a sequence of alternatives which converges to the null one as the sample size increases, that is, by using the so-called local alternatives. These kinds of alternatives has been widely used in the literature to measure how sensitive is a test under small deviations from the null hypothesis.

Suppose that $\mathcal{X}$ is an FRV and that $\sigma_{\mathcal{X}}^{2}=\sigma_{0}^{2} \in \mathbb{R}^{+}$and $E\left(\|s \mathcal{X}\|^{4}\right)<\infty$. Let $\left\{\mathcal{X}_{1}, \ldots, \mathcal{X}_{n}\right\}$ be a simple random sample obtained from the FRV $\mathcal{X}$, and consider a 'correction' $\left\{\mathcal{X}_{1}^{[n]}, \ldots, \mathcal{X}_{n}^{[n]}\right\}$ of $\left\{\mathcal{X}_{1}, \ldots, \mathcal{X}_{n}\right\}$ defined as

$$
\mathcal{X}_{i}^{[n]}=\sqrt{1+\frac{a_{n}}{\sqrt{n}}} \mathcal{X}_{i}, i=1, \ldots, n
$$

in order to obtain FRVs whose variance are

$$
\sigma_{\mathcal{X}_{i}^{[n]}}^{2}=\sigma_{n}^{2}=\left(1+\frac{a_{n}}{\sqrt{n}}\right) \sigma_{0}^{2}
$$

where $a_{n} \in(-1,0) \bigcup(0, \infty)$. Thus, if $\left|a_{n}\right| \nearrow \infty$ and $a_{n} / \sqrt{n} \rightarrow 0$ as $n \rightarrow \infty$, then the sequence of the variances $\left\{\sigma_{n}^{2}\right\}_{n}$ converges to $\sigma_{0}^{2}$ as the sample size $n$ tends to $\infty$. Then, the null hypothesis is not verified, but it is approached as $n$ tends to infinity. In Theorem 6 it will be proved that the power under these local alternatives converges to 1.

Theorem 6 Let $\mathcal{X}$ be an FRV such that $\sigma_{\mathcal{X}}^{2}=\sigma_{0}^{2} \in \mathbb{R}^{+}$and suppose that the previous conditions are satisfied. If the asymptotic testing procedure in Section 3 is applied to the sequence of the corrected samples $\left\{\mathcal{X}_{1}^{[n]}, \ldots, \mathcal{X}_{n}^{[n]}\right\}_{n}$, then

$$
\lim _{n \rightarrow \infty} P\left(\left|T_{n}^{[n]}\right|>t_{(1-\alpha) / 2}\right)=1 .
$$


Proof. Since

$$
\overline{\mathcal{X}_{n}^{[n]}}=\sqrt{1+\frac{a_{n}}{\sqrt{n}}} \overline{\mathcal{X}_{n}}, \quad \widehat{S}_{\mathcal{X}[n]}^{2}=\frac{1}{n-1} \sum_{i=1}^{n} D\left(\mathcal{X}_{i}^{[n]}, \overline{\mathcal{X}_{n}^{[n]}}\right),
$$

and

$$
\widehat{\sigma}_{\mathcal{X}^{[n]}}^{2}=\frac{1}{n} \sum_{i=1}^{n} D\left(\mathcal{X}_{i}^{[n]}, \overline{\mathcal{X}_{n}^{[n]}}\right), \quad \widehat{\sigma}_{D\left(\mathcal{X}^{[n]}, \overline{\mathcal{X}}_{n}^{[n]}\right)}^{2}=\frac{1}{n} \sum_{i=1}^{n}\left(D\left(\mathcal{X}_{i}^{[n]}, \overline{\mathcal{X}_{n}^{[n]}}\right)-\widehat{\sigma}_{\mathcal{X}}^{2[n]}\right)^{2}
$$

the test statistic can be written as

$$
T_{n}^{[n]}=\frac{\sqrt{n}\left(\widehat{S}_{\mathcal{X}[n]}^{2}-\sigma_{0}^{2}\right)}{\sqrt{\left.\widehat{\sigma}_{D(\mathcal{X}}^{2}(n], \overline{\mathcal{X}}_{n}^{[n]}\right)}}=\frac{\sqrt{n}\left[\left(1+\frac{a_{n}}{\sqrt{n}}\right) \widehat{S}_{\mathcal{X}}^{2}-\sigma_{0}^{2}\right]}{\left(1+\frac{a_{n}}{\sqrt{n}}\right) \sqrt{\widehat{\sigma}_{D\left(\mathcal{X}, \overline{\mathcal{X}}_{n}\right)}^{2}}} .
$$

The term $\sigma_{n}^{2}$ can be introduced in this expression, obtaining

$$
T_{n}^{[n]}=\frac{\sqrt{n}\left[\left(1+\frac{a_{n}}{\sqrt{n}}\right) \widehat{S}_{\mathcal{X}}^{2}-\sigma_{n}^{2}\right]}{\left(1+\frac{a_{n}}{\sqrt{n}}\right) \sqrt{\widehat{\sigma}_{D\left(\mathcal{X}, \overline{\mathcal{X}}_{n}\right)}^{2}}}+\frac{\sqrt{n}\left[\sigma_{n}^{2}-\sigma_{0}^{2}\right]}{\left(1+\frac{a_{n}}{\sqrt{n}}\right) \sqrt{\widehat{\sigma}_{D\left(\mathcal{X}, \overline{\mathcal{X}}_{n}\right)}^{2}}} .
$$

The first term converges to a $\mathcal{N}(0,1)$ distribution by using the CLT for UH-statistics. In addition, the conditions $\left|a_{n}\right| \nearrow \infty$ and $a_{n} / \sqrt{n} \rightarrow 0$ as $n \rightarrow \infty$ imply the convergence to $\infty$ of the absolute value of second term. Therefore $\left|T_{n}^{[n]}\right| \rightarrow \infty$, and $\lim _{n \rightarrow \infty} P\left(\left|T_{n}^{[n]}\right|>\right.$ $\left.t_{(1-\alpha) / 2}\right)=1$.

The consistency of the one-side tests can be proved by using analogous arguments that of Theorem 6 . The result is established as follows:

Theorem 7 Let $\mathcal{X}$ be an FRV such that $\sigma_{\mathcal{X}}^{2}=\sigma_{0}^{2} \in \mathbb{R}^{+}$and $E\left(\|s \mathcal{X}\|^{4}\right)<\infty$. For all $n \in \mathbb{N}$, let $\left\{\mathcal{X}_{1}, \ldots, \mathcal{X}_{n}\right\}$ be a simple random sample of $F R V$ s from $\mathcal{X}$. In addition, consider a sequence so that $a_{n} \in(-1, \infty), a_{n} \rightarrow \infty$ and $a_{n} / \sqrt{n} \rightarrow 0$ as $n \rightarrow \infty$.

i) To test $H_{0}: \sigma_{\mathcal{X}}^{2} \leq \sigma_{0}^{2}$ against $H_{1}: \sigma_{\mathcal{X}}^{2}>\sigma_{0}^{2}$, consider a corrected sample $\left\{\mathcal{X}_{1}^{[n]}, \ldots, \mathcal{X}_{n}^{[n]}\right\}$ of $\left\{\mathcal{X}_{1}, \ldots, \mathcal{X}_{n}\right\}$ such that

$$
\mathcal{X}_{i}^{[n]}=\sqrt{1+\frac{a_{n}}{\sqrt{n}}} \mathcal{X}_{i}, i=1, \ldots, n,
$$

(with $\left.\sigma_{\mathcal{X}_{i}^{[n]}}^{2}=\sigma_{n}^{2}=\left(1+a_{n} / \sqrt{n}\right) \sigma_{0}^{2}\right)$. Then, if the asymptotic testing procedure in Section 3 is applied to $\left\{\mathcal{X}_{1}^{[n]}, \ldots, \mathcal{X}_{n}^{[n]}\right\}_{n}$, we have that

$$
\lim _{n \rightarrow \infty} P\left(T_{n}^{[n]}>t_{(1-\alpha)}\right)=1 .
$$


ii) To test $H_{0}: \sigma_{\mathcal{X}}^{2} \geq \sigma_{0}^{2}$ against $H_{1}: \sigma_{\mathcal{X}}^{2}<\sigma_{0}^{2}$, consider a corrected sample $\left\{\mathcal{X}_{1}^{[n]}, \ldots, \mathcal{X}_{n}^{[n]}\right\}$ of $\left\{\mathcal{X}_{1}, \ldots, \mathcal{X}_{n}\right\}$, such that

$$
\mathcal{X}_{i}^{[n]}=\sqrt{1-\frac{a_{n}}{\sqrt{n}}} \mathcal{X}_{i}, i=1, \ldots, n
$$

(with $\left.\sigma_{\mathcal{X}_{i}^{[n]}}^{2}=\sigma_{n}^{2}=\left(1-a_{n} / \sqrt{n}\right) \sigma_{0}^{2}\right)$. Then, if the asymptotic testing procedure in Section 3 is applied to $\left\{\mathcal{X}_{1}^{[n]}, \ldots, \mathcal{X}_{n}^{[n]}\right\}_{n}$, we have that

$$
\lim _{n \rightarrow \infty} P\left(T_{n}^{[n]}<t_{\alpha}\right)=1 \text {. }
$$

Remark 3 Theorem 6 indicates that for any sequence $\left\{a_{n}\right\}_{n}$, s.t. $a_{n} \in(-1,0) \bigcup(0, \infty)$, $\left|a_{n}\right| \nearrow \infty$ and $a_{n} / \sqrt{n} \rightarrow 0$ as $n \rightarrow \infty$, then $\sigma_{n}^{2} \rightarrow \sigma_{0}^{2}$ pointwise, and the asymptotic procedure in Section 3 detects the difference from the null hypothesis a.s. $-[P]$. Theorem 7 indicates the same result for the one-sided tests considering any sequence $\left\{a_{n}\right\}_{n}$, such that $a_{n} \in(-1, \infty), a_{n} \rightarrow \infty$ and $a_{n} / \sqrt{n} \rightarrow 0$ as $n \rightarrow \infty$.

\section{Simulation studies}

In order to empirically justify the use of the tests proposed in this work, some simulation studies have been carried out by following the sample generation procedure developed in [11]. An FRV $\mathcal{X}$ with mean $E(\mathcal{X})=\Pi(-3,-1,1,1.5)$ and variance $\sigma_{\mathcal{X}}^{2}=22.6947$ is simulated. $\Pi(a, b, c, d)$ stands for the well-known $\Pi$-curve, which is defined as the fuzzy set on $\mathbb{R}$ such that for each $\alpha \in[0,1]$ and $a \leq b \leq c \leq d: \Pi(a, b, c, d)_{\alpha}=$ $\left[\inf S(a, b, c)_{\alpha}, \sup Z(b, c, d)_{\alpha}\right]$ with

$$
\begin{aligned}
& S(a, b, c)_{\alpha}= \begin{cases}{[a+(b-a) \sqrt{\alpha / 2}, c]} & \text { if } \alpha \leq .5 \\
{[b+(a-b) \sqrt{(1-\alpha) / 2}, c]} & \text { if } \alpha>.5\end{cases} \\
& Z(a, b, c)_{\alpha}= \begin{cases}{[a, c+(b-c) \sqrt{\alpha / 2}]} & \text { si } \alpha \leq .5 \\
{[a, b+(c-b) \sqrt{(1-\alpha) / 2}]} & \text { if } \alpha>.5\end{cases}
\end{aligned}
$$

In this case, $101 \alpha$-levels are considered, by choosing a uniformly distributed RV in $[-8,8]$ for the 'centers' and two RVs which distributed as a $\chi_{1}^{2}$ for the 'shape'. A random sample from $\mathcal{X}$ is obtained by applying the procedure in [11] $n$ times. To measure the distance between two fuzzy sets the Bertoluzza's metric (a particular $D$ metric) with the Lebesgue measures in $[0,1]$ (see [2]) is employed.

The null hypotheses to be tested are $H_{0}: \sigma_{\mathcal{X}}^{2}=22.6947, H_{0}: \sigma_{\mathcal{X}}^{2} \geq 22.6947$ and $H_{0}: \sigma_{\mathcal{X}}^{2} \leq 22.6947$.

Firstly, the results for the asymptotic case are presented. 10,000 simulations of the asymptotic testing procedures have been carried out (which implies a sample error of .00427 with a confidence of $95 \%$ ). The results for different sample sizes $n$ and significance levels $\beta$ are gathered in Table 1 .

On the other hand, 10,000 simulations of the bootstrap tests have been performed at different significance levels $\beta$ and different sample sizes $n$, and with 1,000 bootstrap replications. The results are showed in Tables 2 and 3 . 
Table 1 Empirical percentage of rejections under $H_{0}$ (asymptotic tests)

\begin{tabular}{c|c|c|c|c|c|c|c|c|c|} 
& \multicolumn{3}{|c|}{$H_{0}: \sigma_{\mathcal{X}}^{2}=6.4437$} & \multicolumn{3}{c|}{$H_{0}: \sigma_{\mathcal{X}}^{2} \geq 6.4437$} & \multicolumn{3}{c|}{$H_{0}: \sigma_{\mathcal{X}}^{2} \leq 6.4437$} \\
\hline$n \backslash 100 \beta$ & 1 & 5 & 10 & 1 & 5 & 10 & 1 & 5 & 10 \\
\hline \hline 50 & 2.02 & 7.04 & 12.32 & 2.94 & 7.86 & 13.28 & .46 & 3.74 & 8.82 \\
\hline 100 & 1.60 & 5.86 & 10.70 & 2.02 & 7.16 & 11.76 & .52 & 4.32 & 9.24 \\
\hline 500 & 1.18 & 5.56 & 10.60 & 1.38 & 5.54 & 10.46 & .86 & 4.75 & 9.74 \\
\hline 1,000 & 1.12 & 5.16 & 10.21 & 1.32 & 5.28 & 9.96 & .89 & 4.86 & 10.06 \\
\hline 5,000 & .96 & 5.08 & 10.04 & 1.12 & 5.01 & 9.99 & .94 & 5.02 & 9.98 \\
\hline
\end{tabular}

Table 2 Empirical percentage of rejections under $H_{0}$ (bootstrap 1)

\begin{tabular}{c|c|c|c|c|c|c|c|c|c|} 
& \multicolumn{3}{|c|}{$H_{0}: \sigma_{\mathcal{X}}^{2}=6.4437$} & \multicolumn{3}{c|}{$H_{0}: \sigma_{\mathcal{X}}^{2} \geq 6.4437$} & \multicolumn{3}{c|}{$H_{0}: \sigma_{\mathcal{X}}^{2} \leq 6.4437$} \\
\hline$n \backslash 100 \beta$ & 1 & 5 & 10 & 1 & 5 & 10 & 1 & 5 & 10 \\
\hline \hline 10 & 2.62 & 8.08 & 13.10 & .42 & 2.34 & 5.18 & 8.68 & 15.84 & 21.92 \\
\hline 30 & 2.08 & 5.44 & 11.44 & .56 & 2.68 & 5.92 & 4.42 & 10.70 & 16.16 \\
\hline 50 & 1.50 & 5.36 & 10.54 & .69 & 3.05 & 6.34 & 3.06 & 8.98 & 14.92 \\
\hline 100 & 1.25 & 5.28 & 9.90 & .78 & 4.12 & 8.19 & 1.83 & 6.27 & 11.84 \\
\hline 200 & 1.09 & 5.06 & 9.95 & .89 & 4.68 & 9.39 & 1.23 & 5.61 & 10.34 \\
\hline
\end{tabular}

Table 3 Empirical percentage of rejections under $H_{0}$ (bootstrap 2)

\begin{tabular}{c|c|c|c|c|c|c|c|c|c|} 
& \multicolumn{3}{|c|}{$H_{0}: \sigma_{\mathcal{X}}^{2}=6.4437$} & \multicolumn{3}{c|}{$H_{0}: \sigma_{\mathcal{X}}^{2} \geq 6.4437$} & \multicolumn{3}{|c|}{$H_{0}: \sigma_{\mathcal{X}}^{2} \leq 6.4437$} \\
\hline$n \backslash 100 \beta$ & 1 & 5 & 10 & 1 & 5 & 10 & 1 & 5 & 10 \\
\hline \hline 10 & .73 & 3.62 & 7.82 & .44 & 2.84 & 5.72 & 1.49 & 8.62 & 16.22 \\
\hline 30 & .80 & 4.07 & 9.12 & .64 & 3.62 & 6.84 & 1.32 & 6.78 & 13.92 \\
\hline 50 & .88 & 4.38 & 9.46 & .70 & 3.95 & 8.26 & 1.26 & 6.14 & 12.52 \\
\hline 100 & .92 & 4.88 & 9.61 & .84 & 4.36 & 8.71 & 1.17 & 5.52 & 10.16 \\
\hline 200 & .98 & 4.95 & 9.82 & .93 & 4.89 & 10.87 & 1.08 & 5.23 & 10.09 \\
\hline
\end{tabular}

Table 1 shows that only when $n \geq 1,000$, the empirical percentage of rejections is quite close to the nominal significance level, which implies that the asymptotic test requires large samples. However, the results of both bootstrap tests are quite good from $n \geq 50$ as show Tables 2 and 3, especially those of the second bootstrap.

On the other hand, some simulations of a triangular-valued FRV $\mathcal{X}$ are carried out to show the consistency of the proposed tests. Specifically, the 'center' (or vertex) of the fuzzy numbers is given by a $\mathcal{N}(1,2)$ variable and the left and right spreads have $\chi_{3}^{2}$ and $\chi_{8}^{2}$ distributions, respectively. In this setting, the variance of $\mathcal{X}$ is assumed to be $\sigma_{\mathcal{X}}^{2}=6.4437$.

Firstly, the power of the tests $H_{0}: \sigma_{\mathcal{X}}^{2} \leq 6.4437$ and $H_{0}: \sigma_{\mathcal{X}}^{2} \geq 6.4437$ is analyzed. For this purpose, the alternative hypotheses $\sigma^{2}=\left(1-\frac{1}{m}\right) \sigma_{0}^{2}$ and $\sigma^{2}=\left(1+\frac{1}{m}\right) \sigma_{0}^{2}$ (with $m$ a positive real number) are considered respectively, where $\sigma_{0}^{2}=6.4437$. The power function of both tests at the level $\alpha=.05$ is shown in Figure 1. Ten thousand simulations of the test have been carried out, using a sample size of one hundred and for different values of $m$. As a result, Figure 1 shows that the power is close to 1 as far as the alternative hypothesis is from the null one in both cases

In the same way, to analyze the power of the test $H_{0}: \sigma_{\mathcal{X}}^{2}=6.4437$, the alternative hypotheses $\sigma^{2}=\left(1+\frac{1}{m}\right) \sigma_{0}^{2}$ are considered. Figure 2 shows that the power function of the two-sided test at the level $\alpha=.05$, constructed under the same conditions as in the one-sided case, is close to 1 as far as the alternative hypothesis is from the null one. 
Fig. 1 Power of the tests $H_{0}: \sigma_{\mathcal{X}}^{2} \leq 6.4437$ and $H_{0}: \sigma_{\mathcal{X}}^{2} \geq 6.4437$
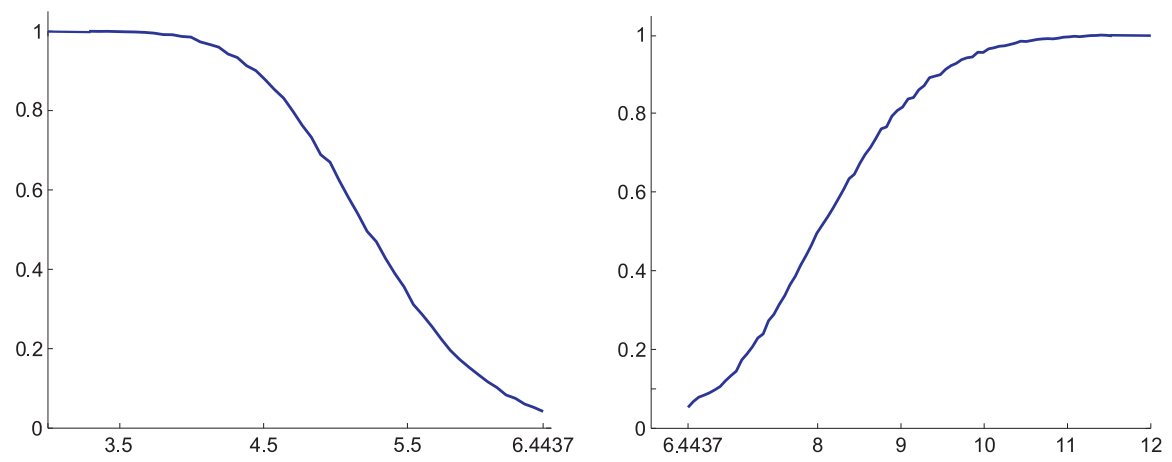

Fig. 2 Power of the test $H_{0}: \sigma_{\mathcal{X}}^{2}=6.4437$

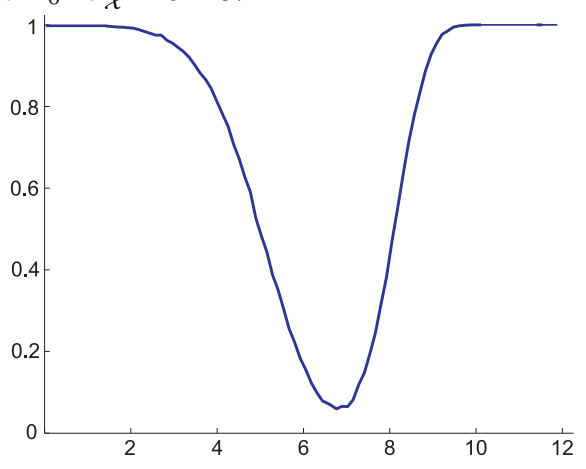

\section{Illustrative examples}

The results obtained in this paper are applied in this section to a real-life example inspired by [7]. The decision problem of investing in the stock exchange is analyzed. Suppose that an investor is willing to invest in the stock exchange and that the investor knows the prediction of 20 experts about the index at the end of 2007. The experts opinions are not given by a single value, but by three values: two of them represent the limits of the interval where they think the index value will be included for sure, and the third one represents the maximum presumption they have about the value of the index. These opinions could be represented by means of triangular fuzzy numbers and they are gathered in Table 4.

Example 1. Suppose that the investor is not too risky and that he/she will only invest if the expert opinions have a variability of 500 points at most. In order to advise the investor, the random sample from $\mathcal{X} \equiv$ index at the end of 2007 given by the data in Table 4 is considered, and the test $H_{0}: \sigma_{X} \leq 500$ against $H_{1}: \sigma_{X}>500$ is proposed (or, equivalently, $H_{0}: \sigma_{X}^{2} \leq 250,000$ against $H_{1}: \sigma_{X}^{2}>250,000$ ).

The asymptotic and bootstrap techniques developed in this paper are applied. Using the sample data, $\widehat{S}_{\mathcal{X}}^{2}=551,953.2164$ and the statistic value is $T_{n}=2.5185$. Therefore, 
Table 4 Evolution perspectives of the index

\begin{tabular}{|l|l|c|c||l|l|c|c|}
\hline Expert & Min & $\begin{array}{c}\text { Max } \\
\text { presump. }\end{array}$ & Max & Expert & Min & $\begin{array}{c}\text { Max } \\
\text { presump. }\end{array}$ & Max \\
\hline 1 & 6500 & 8200 & 9000 & 11 & 6500 & 8000 & 9500 \\
\hline 2 & 6800 & 7200 & 8000 & 12 & 6800 & 8500 & 10500 \\
\hline 3 & 6600 & 7000 & 7800 & 13 & 7500 & 9000 & 10200 \\
\hline 4 & 5000 & 7400 & 8000 & 14 & 5500 & 7800 & 9200 \\
\hline 5 & 6300 & 6700 & 7900 & 15 & 7100 & 8200 & 8500 \\
\hline 6 & 5800 & 8000 & 8200 & 16 & 7300 & 8000 & 9800 \\
\hline 7 & 6800 & 9000 & 10000 & 17 & 7800 & 8200 & 9000 \\
\hline 8 & 7000 & 8200 & 9500 & 18 & 8000 & 9300 & 10300 \\
\hline 9 & 5000 & 6500 & 8200 & 19 & 7200 & 8500 & 9400 \\
\hline 10 & 6800 & 7900 & 8500 & 20 & 6900 & 7000 & 8800 \\
\hline
\end{tabular}

the asymptotic test leads to a $p$-value of .0059 . The first bootstrap tests with 10,000 bootstrap replications leads a $p$-value approximately 0 and the second one to a $p$-value of .0006. So, in any case the conclusion is that the investor should not invest his capital in the stock exchange at the usual significance levels.

Example 2. Under the same conditions than the previous example, suppose now that the investor decides to be riskier and he/she will invest if the expert opinions have a variability of 700 points at most. Then, the aim is to test $H_{0}: \sigma_{X} \leq 700$ (or $\left.H_{0}: \sigma_{X}^{2} \leq 490000\right)$. The statistic value in this case is $T_{n}=.5161$. The $p$-value of the asymptotic test is .3029 , and the corresponding to the bootstrap tests with 10000 replications are .21498 and .22263 respectively, so the investor could invest his capital at the usual significance levels.

\section{Conclusions and open problems}

In this paper a test for the variance of an FRV has been developed. The concept of variance considered here is based on a generalized metric and it is employed to quantify the variability of the fuzzy values of an FRV about its expected value. The developments were based on some classical results for Hilbertian random variables. This has been possible thanks to the employment of the support function, which is a very useful tool for theoretical developments.

Asymptotic and bootstrap procedures have been analyzed. All the test are asymptotically correct and the analysis of local alternatives leads to similar conclusions than the corresponding ones in the real-valued case. The simulations showed that the bootstrap techniques are suitable for small and moderate sample sizes.

The main advantage of the results in this work w.r.t. the existing procedures in the literature (see [16]) is that the techniques employed here are valid for any type of FRVs with values in $\mathcal{F}_{c}\left(\mathbb{R}^{p}\right)$, and not only for FRV taking on a finite number of different values in $\mathcal{F}_{c}(\mathbb{R})$. In addition, some power studies supporting the suitability of the tests have been established.

In the future, the problem of testing the equality of variances of two or more FRVs can be carried out in order to analyze the homoscedasticity condition.

Acknowledgements The research in this paper has been partially supported by the Spanish Ministry of Education and Science Grants MTM2006-07501 and by the European Centre for Soft Computing. 


\section{References}

1. Aumann RJ (1965) Integrals of set-valued functions. J. Math. Anal. Appl. 12:1-12

2. Bertoluzza C, Corral N, Salas A (1995) On a new class of distances between fuzzy numbers. Mathware \& Soft Computing 2:71-84

3. Bickel PJ, Freedman DA (1981) Some asymptotic theory for the bootstrap. The Annal of Statistics 9, No. 6:1196-1217

4. Billingsley P (1986) Probability and measure. John Wiley \& Sons, Inc., Chicago

5. Borovskikh YuV (1986) Theory of U-statistics in Hilbert spaces. Preprint, No. 86.78. UkrSSR Academy of Sciences, Institute of Mathematics, Kiev

6. Colubi A, Domínguez-Menchero JS, López-Díaz M, Ralescu R (2002) A $D_{E}[0,1]$ representation of random upper semicontinuous functions. Proc. Amer. Math. Soc. 130:3237-3242

7. Corbatón JA, Ceballos D (2004) Aplicación del método Fuzzy Delphi a la predicción bursátil. XI Congress of International Association for Fuzzy-set management and Economy, University Mediterranea of Reggio Calabria (Italia)

8. Diamond P, Kloeden P (1994) Metric Spaces of Fuzzy Sets. World Scientific, Singapore

9. Fréchet M (1948) Les éléments aléatoires de nature quelconque dan un espace distancié. Ann. Inst. Henri Poincaré 10:215-310

10. Gil MA, Montenegro M, González-Rodríguez G, Colubi A, Casals MR (2006) Bootstrap approach to the multi-sample test of means with imprecise data. Comput. Statist. Data Anal. $51: 148-162$

11. González-Rodríguez G, Colubi A, Trutschnig W (2008) Simulating random upper semicontinuous functions: the case of fuzzy data. Submitted

12. González-Rodríguez G, Montenegro M, Colubi A, Gil MA, (2006) Bootstrap techniques and fuzzy random variables: Synergy in hypothesis testing with fuzzy data. Fuzzy Sets and Systems 157:2608-2613

13. Hoeffding W (1948) A Class of Statistics with Asymptotically Normal Distribution. The Annal of Mathematical Statistics 19:293-325

14. Körner R (2000) An asymptotic $\alpha$-test for the expectation of random fuzzy variables. J. Stat. Plann. Inference 83:331-346

15. Kratschmer V (2001) A unified approach to fuzzy random variables. Fuzzy Sets and Systems 123:1-9

16. Lubiano MA, Alonso C, Gil MA (1999) Statistical inferences on the S-mean squared dispersion of a fuzzy random variable. In: Proceedings of the Joint EUROFUSE-SIC99, Budapest, pp. $532-537$

17. Montenegro M, Colubi A, Casals MR, Gil MA (2004) Asymptotic and Bootstrap techniques for testing the expected value of a fuzzy random variable. Metrika 59:31-49

18. Näther W (2000) On random fuzzy variables of second order and their application to linear statistical inference with fuzzy data. Metrika 51:201-221

19. Näther W, Wünche A (2007) On the conditional variance of fuzzy random variables. Metrika 65:109-122

20. Puri M, Ralescu DA (1985) The Concept of Normality for Fuzzy Random Variables. The Annals of Probability 13:1373-1379

21. Puri ML, Ralescu DA (1986) Fuzzy random variables. J. Math Anal. Appl. 114:409-422

22. Zadeh LA (1975) The concept of a linguistic variable and its application to approximate reasoning. Part 1. Inform. Sci. 8:199-249; part 2. Inform. Sci. 8:301-353; part 3. it Inform. Sci. 9:43-80 